\title{
Regular Article \\ Mental illness in a general hospital's family medicine clinic in Taiwan
}

\author{
CHIA YIH LIU, MD, ${ }^{1}$ CHING YU CHEN, MD $^{2}$ AND ANDREW T.A. CHENG, MD, $\mathrm{PhD}^{3}$ \\ ${ }^{1}$ Department of Psychiatry, Chang Gung Memorial Hospital and Chang Gung University School of Medicine, \\ Taoyuan, ${ }^{2}$ Department of Family Medicine, National Taiwan University Hospital and National Taiwan \\ University College of Medicine and ${ }^{3}$ Institute of Biomedical Science, Academia Sinica, Taipei, Taiwan
}

\begin{abstract}
There has been a paucity of evidence-based information regarding mental illness in general medical settings among the Chinese population. Aims of the present study are to investigate the patterns and distribution of mental disorders among family medicine clinic attenders, illness behavior of attenders with such disorders, and the recognition and management given by family physicians for them. A random sample $(n=200)$ of patients attending a family medicine clinic in a medical center in Taiwan, received a standardized psychiatric assessment using the semistructured Clinical Interview Schedule (CIS) after they had seen the family physician. A total of 12 family physicians, who were blind to the psychiatric status of their patients, were then asked to assess the physical and psychological condition of the subjects. Of all study subjects, $51 \%$ had at least one psychiatric diagnosis based on the CIS assessment, and the corresponding figure was $21.5 \%$ from family physicians. While the specificity of psychiatric case identification among family physician was high $(91 \%)$, the sensitivity was very low (30\%), especially for depressive disorders and alcohol-related disorders. Because the same magnitude of psychiatric morbidity and similarly low detection rates by general medical physicians were found in other developing countries, the improvement in psychiatric education for medical students and family physicians is highly implicated there.
\end{abstract}

Key words case identification, general health care, mental illness, prevalence.

\section{INTRODUCTION}

Previous studies have demonstrated that mental illness constitutes a major health problem in primary care. ${ }^{1,2}$ In the past three decades, studies of mental disorders in primary care have included the estimation of prevalence, ${ }^{3-6}$ the development of case finding instruments, ${ }^{4,7}$ the investigation over the psychiatric case finding practice of primary care physicians, ${ }^{4,6}$ pathways to psychiatric care ${ }^{8,9}$ classification of mental illness, ${ }^{10}$ the outcome of mental illness in primary care, ${ }^{11,12}$ economic issues of such morbidity, ${ }^{13,14}$ and so forth.

These surveys have shown: that mental disorders are frequent in primary care; that a substantial proportion of these disorders are neither recognized nor ade-

Correspondence address: Professor Andrew T.A. Cheng, Institute of Biomedical Science, Academia Sinica, Taipei, Taiwan. Email: bmandrew@gate.sinica.edu.tw

Received 25 December 2003; accepted 14 March 2004. quately treated, leading to a poor outcome ${ }^{15}$ and that a considerable burden from such morbidity has been imposed on individuals and the community. ${ }^{12,16}$

The majority of the studies in primary care psychiatry have been conducted in western countries, where a well-established primary care system has long existed. There has been a paucity of evidence-based information in this area from developing countries, where relatively different health care delivery systems can be found. ${ }^{8}$ Aims of the present study are to investigate the frequency and distribution of psychiatric morbidity, characteristics of attenders with such morbidity, and the ability of family physician in detecting mental illness in a family medicine clinic in Taiwan.

\section{SUBJECTS AND METHODS}

\section{Study site}

This study was conducted in a family medicine clinic of a medical center in Taipei. The clinic has been run by 
the Department of Family Medicine, National Taiwan University Hospital since 1984. Although situated in Taipei City, a large proportion of the clinic's attenders have come from all parts of Taiwan.

Only those who have never visited any specialist clinic of other departments in the Hospital are permitted to consult this clinic. Those found to have major diseases are then referred to various special clinics for further management.

\section{Study subjects and research strategy}

Every one in two consecutive first-visit attenders of the clinic aged 15 and above, were randomly selected to make up a total of 200 sample subjects. The study subjects, while waiting to see the family physicians, were first interviewed by a research assistant on their general health condition, previous help-seeking pattern, and sociodemographic characteristics. After the family physician had finished the assessment, all study subjects were brought to visit a consultant psychiatrist of the Department for a psychiatric interview. Any psychiatric service indicated was finally provided. In total, 12 consultant family physicians participated in this study.

The family physicians, who were blind to the selection of their patients for a psychiatric assessment, were later asked to assess the physical and psychological conditions of every study subject and the relationship between them. Information concerning psychiatric diagnosis and management given by the family physicians for any study subject was collected.

\section{Case definition and case identification}

A Chinese version of the semistructured version of the Clinical Interview Schedule (CIS), developed by Goldberg and his colleagues, ${ }^{17}$ was applied for case identification. The CIS was primarily designed to specifically focus on psychological symptoms commonly seen in general practice settings and the community. The severity rating for individual CIS symptoms includes: 0 (none); 1 (subclinical); 2 (clinical, mild); 3 (clinical, moderate); and 4 (clinical, marked). It is one of the interviews most commonly used in general practice research. The Chinese version of the CIS has been developed and used in community and general medical settings in Taiwan. ${ }^{18-20}$ The interrater reliability of CIS at item level between British CIS workers and our consultant psychiatrist, as well as between psychiatrists in Taiwan was found to be acceptable. ${ }^{21,22}$ Psychiatric diagnoses based on CIS assessment were made by our consultant psychiatrist according to the ICD-10 Diagnostic Criteria for Research (ICD-10-DCR). ${ }^{23}$

\section{Statistical analyses}

Simple descriptive statistics were used to show the characteristics of responders and the distribution of psychiatric diagnoses in both sexes. To assess the validity of psychiatric diagnosis by family physicians against the psychiatrist's diagnosis, the sensitivity, specificity, and overall misclassification rate were calculated.

\section{RESULTS}

\section{Characteristics of respondents}

All of the sample subjects, including 90 men (45\%) and 110 women $(55 \%)$, were successfully investigated. Their ages ranged from 15 to 86, with a mean of 37.3 years (SD 16.9). Among them, 85 (42.5\%) were single, $98(49.0 \%)$ married, and $17(8.5 \%)$ divorced or widowed. A total of $19(9.5 \%)$ were illiterate, 51 (25.5\%) received primary education (6 years), 68 (34\%) had 912 years middle school education, and $62(31 \%)$ were college graduates or higher. A total of $70 \%$ of the respondents were at work and $30 \%$ were unemployed or retired. The percentages of upper, middle and lower classes among them were $15 \%, 58.5 \%$ and $26.5 \%$, respectively. There was no significant difference over the distribution of age, marital, and socioeconomic status between the sexes. Only $38.5 \%$ of them resided in Taipei City, and the rest included $47.5 \%$ from northern and 14\% from other parts of Taiwan (including 1.5\% from islands).

Another $20 \%$ of the respondents were either found or suspected to have a major physical disease based on the assessment by a senior family physician who reviewed their medical records approximately 1 month later (results of all laboratory tests were available on records). The rest were judged to have been suffering from minor physical illness or somatic symptoms.

\section{Psychiatric diagnosis based on Clinical Interview Schedule assessment}

Table 1 shows the distribution of psychiatric diagnosis based on the CIS assessment carried out by our consultant psychiatrist. A total of 102 subjects (51\% of the total respondents) were found to have at least one ICD-10-DCR psychiatric diagnosis. The most common diagnostic group was the neurotic, stress-related and somatoform disorders (F40-F48), followed by depressive disorders (F30-F39) and non-organic insomnia (F51.0). Men had a significantly higher rate of alcohol use disorders than women, whilst rates of neurotic, stress-related and somatoform disorders and overall morbidity were significantly higher in women than in men. 
Table 1. Psychiatric diagnoses among family medicine clinic attenders in a medical center in Taipei

\begin{tabular}{|c|c|c|c|}
\hline ICD-10-DCR diagnoses & Male $(n=89)$ & Female $(n=111)$ & Total $(n=200)$ \\
\hline Organic anxiety disorder (F06.4) & $1(1.1)$ & $1(0.9)$ & $2(1.0)$ \\
\hline Alcohol use disorders (F10) & $7(7.9)$ & $1(0.9)$ & $8(4.0) 2$ \\
\hline Bipolar affective disorder (F31) & 0 & $1(0.9)$ & $1(0.5)$ \\
\hline Depressive disorders & $8(9.0)$ & $17(15.3)$ & $25(12.5)$ \\
\hline Depressive episode (F32) & $2(2.2)$ & $7(6.3)$ & $9(4.5)$ \\
\hline Persistent mood disorders (F34, F38) & $6(6.7)$ & $11(9.9)$ & $17(8.5)$ \\
\hline Neurotic, stress-related \& somatoform disorders & $24(27.0)$ & $47(42.3)$ & $71(35.5)^{* *}$ \\
\hline Panic disorder (F41.0) & 0 & $4(3.6)$ & $4(2.0)$ \\
\hline Generalized anxiety disorder (F41.1) & $8(9.0)$ & $8(7.2)$ & $16(8.0)$ \\
\hline Mixed anxiety and depressive disorder (F41.2) & $5(5.6)$ & $6(5.4)$ & $11(5.5)$ \\
\hline Anxiety disorder, unspecified (F41.9) & 0 & $8(7.2)$ & $8(4.0)$ \\
\hline Adjustment reaction (F43.2) & $4(4.5)$ & $9(8.1)$ & $13(6.5)$ \\
\hline Dissociative motor disorder (F44.4) & 0 & $1(0.9)$ & $1(0.5)$ \\
\hline Somatoform disorders (F45) & $7(7.9)$ & $12(10.8)$ & $19(9.5)$ \\
\hline Nonorganic insomnia (F51.0) & $4(4.5)$ & $8(7.2)$ & $12(6.0)$ \\
\hline Pathological stealing (F63.2) & $1(1.1)$ & 0 & $1(0.5)$ \\
\hline Personality disorder (F60) & $1(1.1)$ & $2(1.8)$ & $3(1.5)$ \\
\hline Mild mental retardation (F70.0) & $1(1.1)$ & 0 & $1(0.5)$ \\
\hline Total psychiatric morbidity ${ }^{\dagger}$ & $37(44.5)$ & $65(55.5)$ & $102(51.0)^{*}$ \\
\hline
\end{tabular}

'Each patient may have more than one diagnosis.

Sex difference: $* P<0.05 ; * * P<0.02$.

Table 2. Mental disorders diagnosed by family physicians: prevalence rates (\%) by sex

\begin{tabular}{llrr}
\hline Diagnoses & Male & Female & Total \\
\hline Affective psychoses & 0 & $1(0.9)$ & $1(0.5)$ \\
Anxiety disorder & $4(4.4)$ & $12(10.9)$ & $16(8.0)$ \\
Hysteria & 0 & $1(0.9)$ & $1(0.5)$ \\
Neuroses & $8(8.8)$ & $7(6.4)$ & $15(7.5)$ \\
Alcohol dependence & $1(1.1)$ & 0 & $1(0.5)$ \\
Sleep disorders & $2(2.2)$ & $2(1.8)$ & $4(2.0)$ \\
Kleptomania & $1(1.1)$ & 0 & $1(0.5)$ \\
Psychosomatic disorders & $1(1.1)$ & $3(2.7)$ & $4(2.0)$ \\
Total morbidity* & $17(18.9)$ & $26(23.6)$ & $43(21.5)$ \\
\hline
\end{tabular}

$* \chi^{2}$ test between two sexes: non-significant.

\section{Psychiatric diagnosis by family physicians}

The distribution of psychiatric diagnosis given by the family physicians is shown in Table 2 . Formal psychiatric diagnoses were made in $18.9 \%$ of men and $23.6 \%$ of women without any significant difference between them. The most common diagnoses included: anxiety disorder, non-specific neurosis, sleep disorder, and psychosomatic disorder. The former two categories occupied $72 \%$ of all the diagnoses.

\section{Psychiatric diagnosis: family physician versus psychiatrist}

The overall rate of mental disorders diagnosed by family physicians was much lower than that assessed by the psychiatrist using the CIS (21.5\% vs. $51 \%)$. Family physicians only recognized $69 \%$ of all psychiatric cases among the study subjects. While the specificity for any diagnosis of mental disorder by family physicians was very high $(91 \%)$, the sensitivity was quite low $(30 \%)$ 
with an overall classification rate of 53\%. In particular, the sensitivity and specificity for alcohol use disorders were $14 \%$ and $100 \%$, respectively.

None of the 25 respondents with diagnoses of depressive disorders given by our consultant psychiatrist received this diagnosis from family physicians. Among them, 14 (56\%) did not receive any psychiatric diagnosis and the rest were diagnosed as having anxiety disorder $(n=7)$, hysteria $(n=1)$, insomnia $(n=1)$, tension headache $(n=1)$, and kleptomania $(n=1)$ by family physicians. Family physicians also under-diagnosed alcohol use disorder (only 1 in 8 ) and sleep disorder (only 4 in 12).

Of the six normal respondents who were misclassified by family physicians as having psychiatric morbidity, all had subclinical somatic symptoms. Four of them were diagnosed as having psychosomatic disorders (functional gastrointestinal disorder and tension headache), and the rest with 'globus hystericus' and 'neurotic disorder'. These diagnoses were given only by two out of the 12 family physicians, suggesting a personal preference.

\section{Respondents' chief complaints}

The spontaneous chief complaints of respondents before further enquiry by CIS interview, are shown in Table 3. It was found that $89.5 \%$ of respondents gave entirely physical complaints, $3 \%$ gave entirely psychological complaints, and $0.5 \%$ gave both physical and psychological complaints. The rest $(7 \%)$ visited the clinic for various reasons including health screening and premarital counseling. Around half (52.5\%) of the respondents with entirely physical complaints had psychiatric diagnoses from our consultant psychiatrist.

\section{Previous consultation among respondents}

A high proportion of all respondents (76\%) made one or more consultations for the same chief complaint(s) before visiting the family medicine clinic (Table 4). They sought help from various medical professionals (often multiple) including general practitioners, general hospital doctors, Chinese doctors, pharmacists (they were legally allowed to sell a wide range of drugs without physician's prescription except antibiotics and psychotropics), and laboratory technicians (they were allowed to perform various simple laboratory tests without a physician's order). Only two of them, one with bipolar affective disorder and the other with alcohol dependence, had visited psychiatrists before.

Rates of having any consultation were similar between cases and normal. However, cases significantly outnumbered normal in consulting general hospital doctors and Chinese doctors. The mean number of consultation in the last 1 month before visiting the family medicine clinic was also significantly higher in cases than in normal (Table 4). About a quarter of cases had

Table 3. Chief complaints (\%) spontaneously reported by family medicine clinic attenders

\begin{tabular}{lrcc}
\hline & $\begin{array}{c}\text { Male } \\
(n=90)\end{array}$ & $\begin{array}{c}\text { Female } \\
(n=110)\end{array}$ & $\begin{array}{c}\text { Total } \\
(n=200)\end{array}$ \\
\hline Entirely physical & 85.3 & 92.8 & 89.5 \\
Entirely psychological & 5.6 & 0.9 & 3.0 \\
Physical and psychological & 1.1 & 0.0 & 0.5 \\
Miscellaneous reasons $^{\dagger}$ & 7.9 & 6.3 & 7.0 \\
\hline
\end{tabular}

${ }^{\dagger}$ Including health screening and premarital counseling.

Table 4. Mental illness and previous consultations among family medicine clinic attenders

\begin{tabular}{|c|c|c|c|}
\hline & $\begin{array}{c}\text { Normal } \\
n(\%)\end{array}$ & $\begin{array}{l}\text { Cases } \\
n(\%)\end{array}$ & $\begin{array}{c}\text { Total } \\
n(\%)\end{array}$ \\
\hline \multicolumn{4}{|l|}{ Previous consultation $^{\dagger}$} \\
\hline General practitioner (GP) & $34(34.7)$ & $44(43.1)$ & $78(39.0)$ \\
\hline General Hospital & $21(21.4)$ & $37(36.3)$ & $58(29.0)^{*}$ \\
\hline Pharmacist & $17(17.3)$ & $13(12.7)$ & $30(15.0)$ \\
\hline Laboratory technician & $7(7.1)$ & $5(4.9)$ & $12(6.0)$ \\
\hline Chinese doctor/herbs & $11(11.2)$ & $22(21.6)$ & $33(16.5)^{*}$ \\
\hline None & $25(25.5)$ & $23(22.5)$ & $48(24.0)$ \\
\hline Frequency of consultation (mean, s.d) & $1.8(1.2)$ & $2.3(1.9)$ & $2.0(1.6)$ \\
\hline Ever use hypnotics & $4(4.1)$ & $25(24.5)$ & $29(14.5)^{* *}$ \\
\hline Previously & $2(2.0)$ & $9(8.8)$ & $11(5.5)$ \\
\hline Currently & $2(2.0)$ & $16(15.7)$ & $18(9.0)$ \\
\hline Total no. & 98 & 102 & 200 \\
\hline
\end{tabular}

${ }^{\dagger}$ Each respondent may have more than one consultation; $* P<0.05 ; * * P<0.0001$; ${ }^{\star} \mathrm{t}=2.37, P<0.02$ (in the past 1 month). 
used hypnotics before and $15.7 \%$ were current users. The prevalence rates of psychiatric cases among subjects with and without previous consultation(s) were close to each other, being $52.0 \%(79 / 152)$ and $47.9 \%$ (23/48), respectively.

\section{Management from family physicians}

Among the 43 respondents diagnosed as having mental illness by the family physicians, only two (4.7\%, approximately one out of every 20 cases) were referred to see the psychiatrist. One of them had a psychiatric diagnosis of anxiety disorder, and the other had kleptomania comorbid with a depressive episode. Psychotropic prescription was given for $67.4 \%$ of these 43 respondents, including all the six false positives.

Interestingly, 13 respondents not having any psychiatric diagnosis from family physicians (seven psychiatric cases and six normal) received psychotropic prescription from them. Among the seven cases, three were given hypnotics, including two suffering from major physical conditions comorbid with anxiety and one with long-term school pressure. The family physician did not regard their insomnia as a psychiatric problem. The remaining four were given benzodiazepines to temporarily relieve their pain from somatic symptoms that were suspected to have come from physical diseases.

Although physical examination did not find anything abnormal, a series of laboratory tests were arranged for them. Among the six normal, five were given hypnotics for their transient or occasional sleep problem caused by acute physical conditions or excessive work. The last one had a mild chest discomfort and was worried about having a heart condition, which was found in several members of his family. Anxiolytics were prescribed for him.

\section{DISCUSSION}

\section{Methodological consideration}

This study applied a standardized psychiatric interview among a representative sample from consecutive new visitors of a family medicine clinic in a medical center in Taipei. Although it was only conducted in one family medicine clinic, the study subjects came from various parts of Taiwan and most of them suffered from minor physical illness or somatic symptoms. In fact, nearly all the general hospitals (private and public) and medical centers in Taiwan now have family medicine clinics. More than half of our study subjects had consulted them previously, and only about one-third ever consulted general practitioners for the same discomforts.
The wide variety of medical professionals and traditional healers they had consulted before does reflect the general help-seeking behavior of people in Taiwan. Furthermore, rates of common mental disorders were very similar between study subjects with and without previous consultation. Therefore, we believe that findings in this study can reflect the general picture of psychiatric morbidity in general medical care in Taiwan.

\section{Prevalence of mental disorders in general health care across east and west}

The overall prevalence rates of mental illness in general health care previously reported have varied widely. ${ }^{2,24}$ Such differences might have come from differences in case definition and case identification, in sociodemographic characteristics of study subjects, in general health care settings, and in help-seeking patterns of psychiatric cases in different societies.

It is, therefore, more sensible to compare rates of common mental disorders among studies using the same case finding instrument. When such an exercise was made among studies using the semistructured CIS, it was found that rates were largely similar, around $20 \%$ to $35 \%$ in western societies. ${ }^{4,25}$ Moreover, the corresponding rates in non-western countries, including Taiwan, were generally considerably higher, around $45 \%$ to $55 \%,{ }^{26,27}$ with one exception in the United Arab Emirates $(27.6 \%){ }^{28}$

The evidence seems to suggest that the rates of common mental disorders are generally higher in nonwestern general health care. This might be attributable to either higher prevalence rates of mental disorders in the community there, or differences in pathways to psychiatric care between east and west. ${ }^{9}$ The evidence gathered hitherto does not seem to support the former speculation, since comparable rates of specific mental disorders among general population studies using the same case definition and case identification instruments have been observed over the years. ${ }^{29}$

The difference in prevalence of such morbidity among general health care attenders between developed and developing countries is more likely attributable to differential pathways to psychiatric care among psychiatric cases in the community. First, it may be due to differences in illness behavior, that is, psychiatric cases in developing countries tend to visit general medical physicians rather than psychiatrists. Second, there is much less sufficient psychiatric services in developing countries, leading to a higher proportion of psychiatric patients being treated in general health care. Third, the ability to detect psychiatric cases by general medical physicians is poorer in developing countries, leading to an accumulation of such cases in general 
health care. Fourth, there might be a lower rate of referral of psychiatric patients by general medical physicians contributing to the accumulation of patients.

\section{Differences in pathways to psychiatric care}

In western societies with a well-established health care delivery system, the pathway to psychiatric care essentially follows the model proposed by Goldberg and Huxley (1992) with five levels and four filters. ${ }^{9}$ By and large, a psychiatric case in western communities has to pass the second filter (i.e. the general practitioner's recognition) and the third filter (i.e. the general practitioner's decision to refer) to reach the mental health professional. Hence, a higher permeability for the two filters will bring about a lower prevalence of common mental disorders in general health care. In developing countries lacking such a delivery system, psychiatric cases in the community may be referred by traditional healers, general practitioners or general hospital doctors whom they visit first to receive psychiatric care, or they may indeed directly go to see psychiatric specialists. ${ }^{8}$ All these patterns can be found in Taiwan. In any case, the proportion of psychiatric cases treated by psychiatric professionals would be lower than that in developed nations, and the permeability of the second and the third filter in all the non-psychiatric medical settings is presumably equally low.

In this study, family medicine physicians failed to recognize $69 \%$ of the psychiatric cases, a figure compatible to those in previous studies conducted in developing countries. ${ }^{30}$ It might be postulated that the higher prevalence of common mental disorders found in general health care in developing countries has largely come from a much lower permeability of the second filter, resulting in a higher accumulation of 'hidden psychiatric morbidity' in general health care there.

The lower rate of psychiatric case identification in general health care in developing countries might have come from the characteristic illness behavior of the psychiatric cases and the limited psychiatric education for general medical physicians there. It has been reported that a higher proportion of psychiatric cases in developing countries tend to solely report their somatic symptoms (somatization). ${ }^{31}$ In this study, only $3.5 \%$ of the respondents spontaneously reported psychological discomforts as their chief complaints, compared to the much higher rates among their Western counterparts. ${ }^{9}$ If properly enquired, however, emotional symptoms can be detected among these cases. ${ }^{18}$ The much less psychiatric training for medical students and general medical physicians is attributable to the severely limited manpower in mental health and the relative neglect of health administrators and educators on psychiatric training and education. In Taiwan, residents in the department of family medicine have on average only received 2 months of training in clinical psychiatry.

\section{Mental disorders in general health care}

In this study, common mental disorders were found to have comprised the majority of the psychiatric cases identified in general heath care. This was in accordance with findings in previous works. ${ }^{2,5,6}$ Our finding of a high specificity and a low sensitivity of family physician's diagnosis of common mental disorder are similar to other studies. ${ }^{32}$ The low rate of case detection by family physicians has particularly been observed for depressive disorders and alcoholism. In a large-scale study among 1580 family practice patients, American family physicians detect $27.9 \%$ of depressive disorders. ${ }^{16}$ In another study in Seattle, US and Groningen, the Netherlands, the kappa agreement for depression between primary care physician and CIDI diagnosis was only $0.29 .^{33}$ The condition is even worse in developing countries, where general medical physicians had extremely under-recognized depressive disorders and had rarely prescribed antidepressants. ${ }^{8}$ In this study, none of the patients with depressive disorders were detected by family physicians. The very low rate of detecting alcohol-related disorders by family physicians might be partly due to their lack of alertness on alcohol-related problems. It is evident that the lack of training in detecting emotional symptoms through interview is a serious problem among general medical doctors in Taiwan. Furthermore, psychotropics were found to have been either under-used or misused in their practice and nearly no psychosocial intervention was given.

\section{Implications for further studies and prevention}

Previous work has revealed that higher depressive symptom scores in primary care patients were associated with poorer health, functional status and quality of life, and an increased health care use. ${ }^{34}$ Though effective case finding may not directly predict a good outcome of patients with depression, ${ }^{7}$ it is the first step and prerequisite for improving the process of care.

Since it is possible that the rates of common mental disorders in general medical care in developing countries are higher than those in developed countries, the improvement in the detection and management of cases with such morbidity by general medical physicians are highly indicated in the former. To achieve this, a well-integrated education and training program in psychiatry for both undergraduate and postgraduate 
medical personnel, with emphasis on the characteristic illness behavior of psychiatric cases in developing countries, is urgently needed.

\section{ACKNOWLEDGMENT}

The authors wish to express their thanks to the cooperation of the consultant family physicians in this study.

\section{REFERENCES}

1. Druss BG. The mental health/ primary care interface in the United States: history, structure, context. Gen. Hosp. Psychiatry 2002; 24: 197-202.

2. Goldberg D. Epidemiology of mental disorders in primary care settings. Epidemiol. Rev. 1995; 17: 182-190.

3. Shepherd M, Cooper B, Brown AC et al. Psychiatric Illness in General Practice. Oxford University Press, London, 1996.

4. Goldberg D, Blackwell B. Psychiatric illness in general practice: a detailed study using a new method of case identification. BMJ 1970; 2: 439-443.

5. Barrett JE, Barrett JA, Oxman TE, Gerber PD. The prevalence of psychiatric disorders in a primary care practice. Arch. Gen. Psychiatry 1988; 45: 1100-1106.

6. Vazquez-Barquero JL, Carcia J, Simon JA et al. Mental health in primary care. Bri. J. Psychiatry 1997; 170: 529535.

7. Williams JW Jr, Pignone M, Ramirez G, Stellato CP. Identifying depression in primary care: a literature synthesis of case-finding instruments. Gen. Hosp. Psychiatry 2002; 24: 225-237.

8. Gater R, de Almeida E, Sousa B, Barrientos G et al. The pathways to psychiatric care: a cross-cultural study. Psychol. Med. 1991; 21: 761-774.

9. Goldberg D, Huxley P. Common Mental Disorders: a Biosocial Model. Tavistock-Routledge, London, 1992.

10. Jenkins R, Smeeton N, Shepherd M. Classification of mental disorder in primary care. Psychol. Med. Suppl. 1998; 12: 1-59.

11. Seivewright H, Tyrer P, Casey $\mathrm{P}$ et al. A three-year follow-up of psychiatric morbidity in urban and rural primary care. Psychol. Med. 1991; 21: 495-503.

12. Tiemens BG, Ormel J, Simon GC. Occurrence, recognition, and outcome of psychological disorders in primary care. Am. J. Psychiatry 1996; 153: 634-644.

13. Croft-Jeffereys C, Wilkinson G. Estimated costs of neurotic disorder in UK general practice. Psychol. Med. 1985; 19: 549-558.

14. Simon GE, VonKorff M, Barlow W. Health care costs of primary care patients with recognized depression. Arch. Gen. Psychiatry 1995; 52: 850-856.

15. Rost K, Zhang M, Fortney J, Smith J, Coyne J, Smith GR. Persistently poor outcomes of undetected major depression in primary care. Gen. Hosp. Psych. 1998; 20: 12-20.

16. Coyne JC, Schwenk TL, Fecher-Bates S. Nondetection of depression by primary care physician reconsidered. Gen. Hosp. Psych. 1995; 17: 3-12.
17. Goldberg D, Cooper B, Eastwood MR, Kedward HB, Shepherd M. A standardized psychiatric interview for use in community surveys. Br. J. Prevent Soc. Med. 1970; 24: $18-26$.

18. Cheng TA. A community study of minor psychiatric morbidity in Taiwan. Psychol. Med. 1988; 18: 953-968.

19. Cheng ATA, Gau SFG, Chen THH, Chang JC, Chang YT. A 4-year longitudinal study on risk factors for alcoholism. Arch. Gen. Psychiat. 2003 (in press).

20. Chong MY, Wilkinson G. Validation of 30- and 12-item versions of the Chinese Health Questionnaire (CHQ) in patients admitted for general health screening. Psychol. Med. 1989; 19: 495-505.

21. Cheng TA, Williams P, Clare AW. Reliability study of the Clinical Interview Schedule (CIS) between the British and Chinese psychiatrists. Bull. Chinese Soc. Neurol. Psychiatry 1983; 9: 54-55.

22. Chong MY, Cheng TA. Reliability Study of the Clinical Interview Schedule (CIS): The use of community sample. Bull. Chinese Soc. Neurol. Psychiatry 1985; 11: 27-34.

23. World Health Organization. The ICD-10 Classification of Mental and Behavioural Disorders: Diagnostic Criteria for Research. World Health Organization, Geneva, 1993.

24. Ustun B, Sartorius N. Mental Illness in General Health Care: an International Study. John Wiley \& Sons, Chichester, 1955.

25. Skuse D, Williams P. Screening for psychiatric disorder in general practice. Psychol. Med. 1984; 14: 365-377.

26. Sen B, Wilkinson G, Mari JJ. Psychiatric morbidity in primary health care. A two-stage screening procedure in developing countries: choice of instruments and costeffectiveness. Br. J. Psychiatry 1987; 151: 33-38.

27. Mari JJ, Williams P. Minor psychiatric disorder in primary care in Brazil: a pilot study. Psychol. Med. 1984; 14: 223-227.

28. el-Rufaie OE, Absood GH. Minor psychiatric morbidity in primary health care: prevalence, nature and severity. Int. J. Soc. Psychiatry 1993; 39: 159-166.

29. Cheng ATA. Case definition and culture: are people all the same? Br. J. Psychiatry 2001; 179: 1-3.

30. AL-Jaddou H, Malkawi A. Prevalence, recognition and management of mental disorders in primary health care in Northern Jordan. Acta. Psychiatr. Scand. 1997; 96: 3135 .

31. Kleinman A. Neurasthenia and depression: a study of somatization and culture in China. Cult. Med. Psychiatry 1982; 6: 117-190.

32. Jacob KS, Bhugra D, Lloyd KR et al. Common mental disorders, explanatory models and consultation behaviour among Indian women living in the UK. J. Roy. Soc. Med. 1998; 91: 66-71.

33. Tiemens BG, vonKorff M, Lin EHB. Diagnosis of depression by primary care physicians versus a structured diagnostic interview. Gen. Hosp. Psychiatry 1999; 21: 87-96.

34. Herrman H, Patrick DL, Diehr $\mathrm{P}$ et al. Longitudinal investigation of depression outcomes in primary care in six countries: the LIDO study. Psyhol. Med. 2002; 32: 889-902. 